\title{
An image processing pipeline to segment iris for unconstrained cow identification system
}

https://doi.org/10.1515/comp-2019-0010

Received March 9, 2019; accepted July 16, 2019

\begin{abstract}
One of the most evident costs in cow farming is the identification of the animals. Classic identification processes are labour-intensive, prone to human errors and invasive for the animal. An automated alternative is an animal identification based on unique biometric patterns like iris recognition; in this context, correct segmentation of the region of interest becomes of critical importance. This work introduces a bovine iris segmentation pipeline that processes images taken in the wild, extracting the iris region. The solution deals with images taken with a regular visible-light camera in real scenarios, where reflections in the iris and camera flash introduce a high level of noise that makes the segmentation procedure challenging. Traditional segmentation techniques for the human iris are not applicable given the nature of the bovine eye; at this aim, a dataset composed of catalogued images and manually labelled ground truth data of Aberdeen-Angus has been used for the experiments and made publicly available. The unique ID number for each different animal in the dataset is provided, making it suitable for recognition tasks. Segmentation results have been validated with our dataset showing high reliability: with the most pessimistic metric (i.e. intersection over union), a mean score of 0.8957 has been obtained.
\end{abstract}

Keywords: bovine eye, image processing, iris segmentation, pupil segmentation

\footnotetext{
Silvia M. Castro: Departamento de Ciencias e Ingeniería de la Computación, Universidad Nacional del Sur (UNS), Instituto de Ciencias e Ingeniería de la Computación (ICIC UNS - CONICET), Argentina, Consejo Nacional de Investigaciones Científicas y Técnicas (CONICET), Argentina, E-mail: juan.larregui@cs.uns.edu.ar

^Corresponding Author: Dario Cazzato: Interdisciplinary Centre for Security Reliability and Trust (SnT), University of Luxembourg, Luxembourg, dario.cazzato@uni.lu

Silvia M. Castro: Departamento de Ciencias e Ingeniería de la Computación, Universidad Nacional del Sur (UNS), Instituto de Ciencias e Ingeniería de la Computación (ICIC UNS - CONICET), Argentina, E-mail: smc@cs.uns.edu.ar
}

\section{Introduction}

Animal identification is a familiar topic for livestock producers. One of the most evident costs is given to animal identification, thus it is not surprising that the proposal of automatic cow identification has already been investigated since many decades [1].

Over the last years, bovine identification has become a topic of intense worldwide interest in the aftermath of terrorist incidents, outbreaks of bovine spongiform encephalitis (BSE) and, more recently, reports of E. coli contamination in beef [2]. In fact, food could be intentionally contaminated as a terrorist act. The most efficient and effective way of countering all emergencies including food terrorism is through sensible precautions coupled with strong surveillance. Bovine identification is one of the measures to accomplish the mentioned surveillance task. Many producers still routinely use traditional technologies, such as branding, ear notching, tags, and tattoos; however, they are invasive solutions that cannot ensure adequate security or resistance to tampering [3]. Such systems can be duplicated, swapped, and are in general unable to verify the false insurance claims [4]. Moreover, most of these solutions can differentiate an animal between different owners, but cannot distinguish between two animals. The possibility of a less invasive solution for cow identification systems would be of great benefit since it could cut down costs for farm and cattle industry and, at the same time, improve security and guarantee product traceability.

The application of electronic animal ID technologies is a growing trend in livestock production [5]. Radio Frequency Identification (RFID) based solutions have become a common practice in both industry and academia $[6,7]$. Nevertheless, these systems are usually expensive compared to other forms of tracking, they can fail in case of a harsh environment, and have physical limits; furthermore, they represent an invasive solution. The application of RFID in a non-invasive way, e.g. in a neck collar, does not guarantee safety since IDs may get damaged, lost, or voluntarily removed [8]. Secure methods based on DNA have been proposed [9]; their precision is impressive, but this is 
not a cost-effective solution. Furthermore, these systems are slower in providing an answer compared to other technologies.

All the identification process is labour-intensive, it is prone to human errors, and remains invasive for the animal. It is clear that new identification methods are needed. To this end, vision-based systems can provide non-invasive solutions; in the past, such systems used to be complex, with cameras going to frame grabber boards in high-powered computers that were extremely expensive. Nowadays, vision systems are getting smaller, smarter and self-contained. In light of this, it is advantageous to consider a bovine iris recognition system based on the iris' random patterns and other particular attributes that have been shown capable of generating highly unique identification codes. The effectiveness of such solutions strongly relies on the precise identification of the regions of interest (ROIs). In a broad sense, iris segmentation is a critical part of each iris recognition system, because it defines the inner and outer boundaries of the iris region to be used for feature analysis. It is evident that if the iris regions are not correctly segmented, the presence of eyelashes, eyelids, reflections and pupil, would lead to poor recognition performance [10]. Moreover, there is plenty of work demonstrating how, in many contexts, recognition systems driven by high quality segmentation improve overall performance because the recognition system can process only the relevant features instead of features outside of the object of the recognition task [11, 12].

This work introduces a bovine iris segmentation algorithm that processes images taken in the wild and extracts the iris region. The solution deals with real images taken with a regular visible-light camera, where reflections in the bovine iris and camera flash introduced elevated noise that makes the segmentation procedure challenging. Very few works have been proposed in the state of the art; the problem is challenging since solutions must deal with the physical and biological structure of the bovine eye, thus limiting the usage of the numerous valid techniques proposed for the human eye. The proposed solution can be usable and adaptable by any dynamic identification system, since: (i) the iris region on the input images does not need to be necessarily centred; (ii) the iris region does not need to cover most of the image; (iii) the input images can be captured using a regular visible-light camera on a farm; (iv) both iris and pupil are segmented; (v) the proposed dataset is unique in its typology, considering the lack of data in the state of the art. We think that having an available set of extra information could be fundamental for machine learning based (or other state of the art) recognition systems; thus, the dataset has been designed also for recognition tasks $[13,14]$, providing ground truth information of the iris region and a unique ID number for each different animal in the dataset (see Section 5).

The manuscript is organized as follows: in Section 2 the related state of the art is presented; although there are not existing works dealing with the presented scenario, the section introduces the segmentation first, and then the eye segmentation problems, analyzing solutions about the latter that have been presented in comparable circumstances or inputs. Section 3 introduces the techniques and concepts used by the proposed solution (Section 4). Section 5 gives details of our BovineAAEyes 80 dataset. Experimental setup and results are shown in Sections 6 and 7, respectively. Conclusions and future work are given in Section 8.

\section{State of the art}

Segmentation is among the most fundamental problems in computer vision [12]. It consists of separating an image in disjoint different regions with a special meaning based on image intensity, colour or geometric properties. Ming [15] proposes to divide image engineering into three levels: image processing, analysis and understanding. With this in mind, image segmentation becomes the key step from image processing to image analysis. A typical block diagram of an image-based recognition system is shown in Fig. 1; many works that have been proposed in the literature for animal identification follow this pipeline for achieving recognition [16, 17]. First of all, the input image is acquired, e.g. from IR, RGB, monocular, depth sensors. The image is usually noisy and has many redundant or unnecessary data, thus some pre-processing is often performed to enhance relevant parts and reduce noise. Techniques of image enhancement, colour space transformation and morphological operations are often employed at this aim. The new image is the input for the segmentation step, in order to separate the dominant part for the recognition task from the background. The foreground is used to extract the features needed for the classification task. Note that one or more blocks could be missing; for example, many solutions can directly demand a machine learning method to obtain the identification without having to extract handcrafted features; moreover, direct end-to-end systems have also been recently proposed [18], although in contexts that are different from the one under consideration.

Generally speaking, human authentication based on iris patterns is one of the most popular applications in 


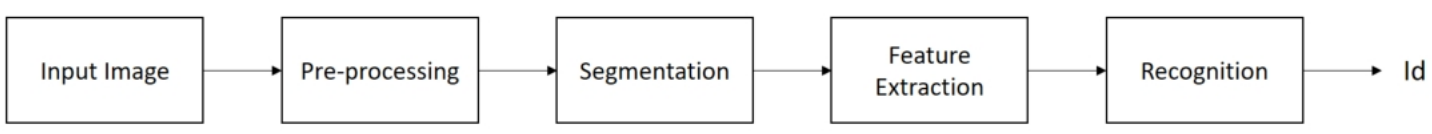

Figure 1: A block diagram of a typical computer vision based identification system.

video and image processing [19]. Such systems rely on the segmentation of the iris region [20-23], i.e. the problem of separating the iris region from the pupil and the sclera. As a consequence, the problem of eye segmentation is a very hot topic in the computer vision community, as can be observed from the state of the art [24-31]. Human iris boundaries are approximately defined by two circles, i.e. the pupillary boundary that divides the pupil and the iris, and the limbic boundary separating the iris and the sclera. Despite active research, eye detection and tracking remain very challenging tasks due to several unique issues including occlusion of the eye by the eyelids, eye open/closed, variability in size, reflectivity, illumination changes, etc. [32]. The complexity is such that even the sub-problems of separating the sclera from the iris or identifying eye regions in human faces became independent research lines [33]. The taxonomy of eye detection techniques mainly consists of feature-based and appearancebased methods. Appearance-based methods make use of the global appearance of the eye [34, 35], while featurebased methods make use of local image features [36-38]. Even hybrid methods that combine the aforementioned solutions have been proposed [39]. Although the majority of approaches cannot be compared with the scope of our manuscript since they are based on the search of circularity patterns or the analysis of the human face, other works have proposed more generic schemes using approaches based on oriented histograms [40], Monte Carlo sampling framework [41], or Convolutional Neural Networks (CNN) [42]. Such works show the importance of computer vision techniques to address the problem under consideration.

If human pupil detection, iris segmentation and/or identification present a very large state of the art, very few works address the problem of image-based bovine iris segmentation, exploring instead uniqueness and invariability of the retinal vascular pattern of the eye [43], making retinal vascular patterns one of the most employed biometric features for identification of cattle [44]. Existing works employ multiple cameras properly installed on a dairy farm [45] or ad-hoc devices made by an optical system, an illumination source, and a viewing screen [46]. Other works tend to be outdated.
Another example of investigated biometrics is muzzle $[44,47]$. In general, these solutions are based on a machine learning scheme applied to previously extracted features using methods like SIFT [48], Weber Local Descriptor [49] or Local Binary Pattern [50]. To this end, interesting work has been proposed in [51]. Authors employ an algorithm to extract biometric features and use them to train a machine learning model, obtaining high performance rates. Nevertheless, best classification rates are achieved using a machine learning solution trained with at most 7 images for each animal, suggesting the need for further studies against a larger database of cattle images.

An algorithm to support bovine iris segmentation based on geometrical features of the cow's eye has been proposed in 2009 by Zhang et al. [52]. In this work, the iris region is approximated using a Sobel edge detection operator, and the coordinates of boundary points are determined via quadratic B-spline interpolation curves. Next, two circles are created which do not exactly delimit the pupil and the iris of the cow but instead isolate part of the iris. Thus, the work proposes a localization algorithm but does not completely solve the segmentation problem. In [53], the iris is segmented by using a region-based active contour model. The work proposes a full pipeline for iris recognition, but the segmentation module only consists of static image intensities extraction. Zhao et al. [54] propose a recognition method which uses a similar iris localization, adding a feature extraction block that employs 2D Gabor filters and a matching test made by a set of Boolean XORs. However, iris images are acquired with specialized hardware that considerably simplifies the segmentation procedure. The work of [55] extracts the inner and outer boundaries of the cow's iris with a segmentation procedure based on ellipse fitting. Finally, a 2D complex wavelet transform (2D-CWT) is used to extract local and global characteristics, encoding the phase of the filtered iris as its features. Results are remarkable, but no dataset is publicly available. This becomes particularly relevant since images have been taken with an ad-hoc device [56]; moreover, the metric used to evaluate the system is based only on ellipse fitting capabilities. In the context of full recognition systems, the work of Larregui et al. [57] tries to put a milestone by proposing a complete solution in a research work 
that considers both segmentation and classification problems. Authors claim to reach a segmentation performance of $91.67 \%$ and an animal recognition accuracy of $85.71 \%$. However, the segmentation accuracy was computed as the number of images correctly segmented over the total number of images, where the decision of the correctness of the segmentation was made qualitatively, without comparing segmentation results with ground truth data.

\section{Preliminaries}

An overview of the techniques, algorithms and methods used in the design of the proposed solution is provided in this section.

\subsection{HSV colour space}

A colour space is a system for the representation of colour in an image. Different from the RGB colour model, which uses the addition of Red, Green and Blue values to represent colour, the HSV colour space represents pixels as Hue, Saturation and Value (or Brightness), and has been already applied in image segmentation contexts $[58,59]$.

To convert from RGB to HSV, the $R, G, B$ values are first normalized as $R^{\prime}, G^{\prime}, B^{\prime}$ to be in the range $[0,1]$.

Let $C_{\min }, C_{\max }, \Delta$ be defined as:

$$
\begin{array}{r}
C_{\min }=\min \left(R^{\prime}, G^{\prime}, B^{\prime}\right) \\
C_{\max }=\max \left(R^{\prime}, G^{\prime}, B^{\prime}\right) \\
\Delta=C_{\max }-C_{\min }
\end{array}
$$

Then, we express HSV values as:

$$
\begin{gathered}
H= \begin{cases}0^{\circ} & \text { if } \Delta=0 \\
60^{\circ}\left(\frac{G^{\prime}-B^{\prime}}{\Delta} \bmod 6\right) & \text { if } C_{\max }=R^{\prime} \\
60^{\circ}\left(\frac{B^{\prime}-R^{\prime}}{\Delta}+2\right) & \text { if } C_{\max }=G^{\prime} \\
60^{\circ}\left(\frac{R^{\prime}-G^{\prime}}{\Delta}+4\right) & \text { if } C_{\max }=B^{\prime}\end{cases} \\
S= \begin{cases}0^{\circ} & \text { if } C_{\max }=0 \\
\frac{\Delta}{C_{\max }} & \text { if } C_{\max } \neq 0\end{cases} \\
V=C_{\max }
\end{gathered}
$$

\subsection{Morphological operations and minima imposition}

Morphological operations process images based on shapes, extracting structure information. For this, the image is probed by a known shape called structuring element (SE). The simplest form of SE is called elementary isotropic structuring element: centred at a pixel $p$, this form corresponds to the neighbours of the pixel $p$ plus the pixel $p$ itself. The neighbours of a pixel depend on the type of pixel connectivity considered. In this paper we use 8-connected pixels, that is, the neighbours are those pixels that are connected horizontally, vertically, and diagonally.

\subsubsection{Erosion and geodesic erosion}

Erosion is one of the fundamental morphological operators, along with dilation. For a binary or grayscale image $I$, the eroded value at a given pixel $p$ is the minimum value of the image in the window defined by the structuring element $B$ centred at $p$ :

$$
\left[\epsilon_{B}(I)\right](p)=\min _{b \in B} I(p+b)
$$

Another type of image transformations is geodesic transformations. Diversely from the mentioned morphological transformations, which take one image and a structuring element as inputs, geodesic transformations take two input images: a marker image and a mask image. For geodesic erosion, specifically, the marker image is eroded by the elementary isotropic structuring element and then forced to remain above the mask image. The mask image imposes a limit to the propagation of the erosion on the marker image. Formally, the geodesic erosion of the marker image $m$ with respect to the mask image $I$ is defined as the pixel-wise maximum between the mask image and the elementary erosion $\epsilon^{(1)}$ of the marker image. For a given pixel $p$ :

$$
\left[\epsilon_{I}^{(1)}(m)\right](p)=\max \left(\left[\epsilon^{(1)}(m)\right](p), I(p)\right)
$$

\subsubsection{Morphological reconstruction}

Morphological Reconstruction of a mask image from a marker image is based on the iteration of geodesic transformations until convergence. Morphological reconstruction by erosion, specifically, is defined as the geodesic erosion of the marker image $m$ by means of a mask image $I$ it- 


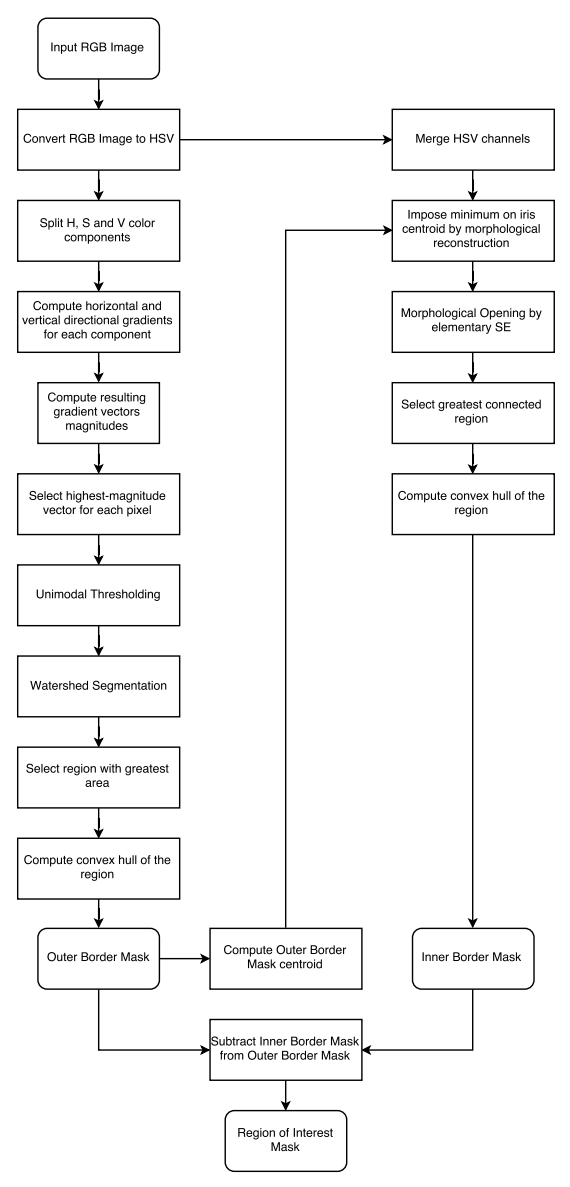

Figure 2: Flowchart algorithm of the proposed solution. Both outer and inner border are extracted on an HSV representation of the input image. The iris region is computed from the magnitudes of the gradients in each pixel, followed by watershed segmentation. For the pupil region, a minimum is imposed in the centroid of the iris mask by morphological minima imposition, and the greatest connected area is considered as the pupil region. The convex hull of both segmented regions constitute the final masks.

erated until stability, that is, until further geodesic erosion does not modify the eroded marker image anymore:

$$
R_{I}^{\epsilon}(m)=\epsilon_{I}^{(i)}(m)
$$

where $i$ represents the iteration number such that $\epsilon_{I}^{(i)}(m)=$ $\epsilon_{I}^{(i+1)}(m)$.

The algorithm always converges after a finite number of iterations for images with bounded intensity levels.

\subsubsection{Regional minima imposition}

A regional minimum is a connected component of pixels with equal intensity values and whose external boundary pixels have all a greater value. The minima imposition operation transforms a grayscale image in such a way that the only remaining regional minima after the transformation are those in previously specified locations [60]. A bi- nary image, called marker image, is created indicating the points or regions where we want the regional minima to be located, assigning a 0 for a pixel $p$ if it belongs to a marker, and 1 otherwise.

The marker image $m$ is defined as:

$$
m(p)= \begin{cases}0, & \text { if } p \text { belongs to a marker, } \\ 1, & \text { otherwise }\end{cases}
$$

The operation is usually implemented using morphological reconstruction by erosion. First, the pixel-wise minimum between the marker $m$ and the input image $I$ is computed, creating minima at locations where the marker image is 0 . This image is then used as a mask image for morphological reconstruction by erosion, employing $m$ as a marker image.

We used a minima imposition operation based on the fast hybrid grayscale morphological reconstruction algorithm described in [61]. 


\subsection{Watershed transform}

The Watershed Transform is a morphological method that segments an image treating it as a topographic map, considering high/low valued pixels in the image as high/low elevations in the map, respectively. The algorithm searches for the regions between the ridges of the surface, called Catchment Basins. A Catchment Basin $C(M)$ associated to a local minimum $M$ is the set of pixels $p$ such that a water drop falling at $p$ flows down on the terrain represented by the image, following a certain descending path, and eventually reaches $M$. The Watershed is defined by the lines that separate adjacent catchment basins.

We based our method in Meyer's variant of the Watershed Transform [62].

\section{Proposed method}

The proposed solution is based on an image processing pipeline that takes as input an RGB image taken with a regular camera. Unlike the majority of state of the art works, we do not use any constraint that helps to identify the region of interest (ROI) by anthropometric relations, like in [63], since a face detector cannot be employed. Moreover, no constraints are given in terms of the position of the eye in the image nor we assume the central pixel being part of the eye region, forcing the algorithm to process all of the pixels in the image. In Figure 2, a flowchart of the algorithm is shown. As in [55], our solution aims at segmenting both outer and inner borders of the image of a bovine open eye, generating a mask of the segmented region after two stages. The outputted ROI is the region between these two boundaries (see Figure 3).

Both stages are based on an HSV representation of the input image. The outer border segmentation stage searches for the highest magnitude gradient in the HSV channels, in each pixel position, to accentuate the frontier, followed by a watershed segmentation of the resulting matrix. The region with the greatest area is considered as the iris region, and its mask is generated as its convex hull. Once this stage is completed, we impose a regional minimum in the centroid of the iris region, which lies inside the pupil region. A morphological opening is then applied, and the connected region with the greatest area is selected as the pupil candidate, defining its convex hull as the pupil mask.
The method assumes the eye to be open, with a visible sclera, as this is the case encountered in practice, except when the bovine blinks.

\subsection{Iris outer border segmentation}

First of all, the original RGB image containing the full visible open eye of the bovine is converted to the HSV colour space (Figures 3a and 4). This colour space highlights the inner and outer boundaries of the iris, due to the differences in pixel intensities of the regions of the cow's eye, in particular in the saturation (S) and value (V) channels. This stems from the fact that the sclera is naturally a low saturation region, whereas the iris region presents high values in the Saturation channel. Moreover, the HSV colour space separates Hue from Saturation, which permits the subsequent steps of the algorithm to be agnostic with respect to the differences in hair, iris and pupil colours between images.

For each of the three resulting channels, the horizontal and vertical directional gradients are computed for each pixel together with the magnitude of the resulting gradient vector. Then, for each pixel, we compare the magnitude of the gradient in the three channels, storing the maximum value in a new matrix. Considering that colour information alone can retain semantics only up to a certain degree [59], the proposed comparison between channels aims to give robustness to the algorithm, taking into account both the colour and lighting changes present in the boundary between the iris and the sclera. At this point, thresholding is applied to the resulting matrix in order to remove noise and background information. This way, only the high magnitude gradients in the image, which are related to borders and high frequency regions, will be kept. Considering that the distribution of values in this image can be approximated to a right-skewed unimodal distribution (see Figure 5), with mode located at the lower end of the histogram, Unimodal Thresholding, a method for bilevel thresholding [64], has been employed.

The Watershed transform is then applied to the output of the previous operation in order to obtain the region of the image surrounded by the iris outer border. In particular, the region with the greatest area in terms of the number of pixels among all of the segmented regions is labelled as ROI.

Last outer border segmentation step aims at removing any imperfections and filter noise by computing the convex hull of the region, leveraging the convex nature of the cow's iris. The output is then used to create a pixel binary 


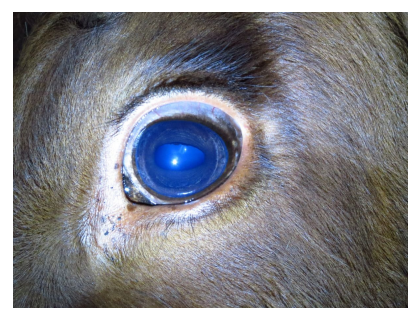

(a)

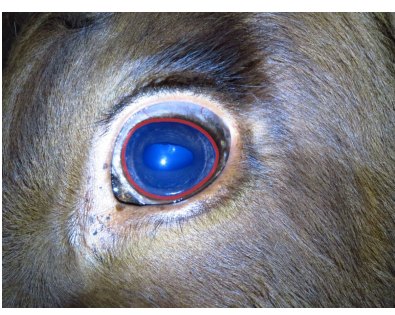

(b)

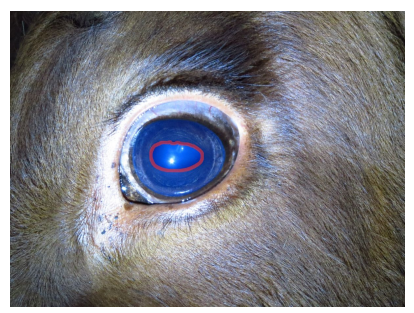

(c)

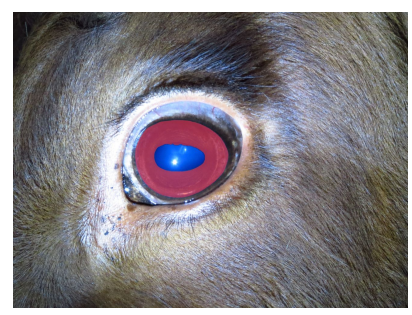

(d)

Figure 3: (a) Original RGB Image, (b) Outer Border, (c) Inner Border, (d) ROI.

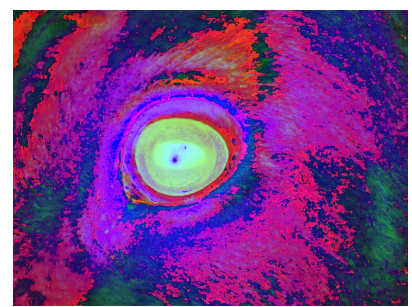

(a)

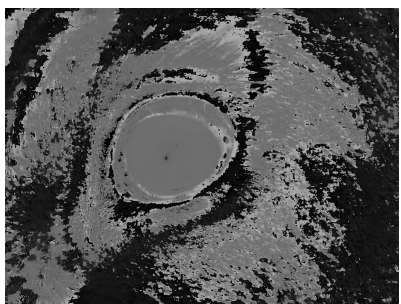

(b)

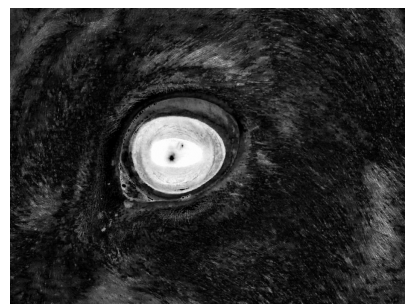

(c)

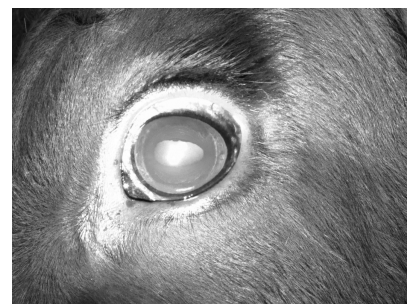

(d)

Figure 4: (a) HSV Image, (b) Hue Channel, (c) Saturation Channel, (d) Value Channel.

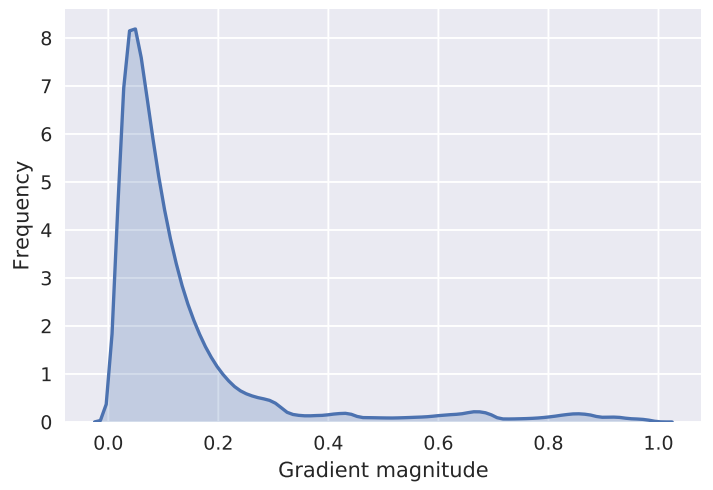

Figure 5: Right-skewed unimodal distribution of gradient magnitudes.

mask that separates the pixels lying inside and outside the iris outer border.

\subsection{Iris inner border segmentation}

The inner border represents the frontier between the iris and the pupil.

We use the same input in the HSV colour space. At this point, we can leverage the knowledge of the iris location obtained from the previous outer border segmentation stage and assume the centroid of the iris mask to be inside the pupil region. This assumption is fulfilled for all the images in the dataset presented in Section 5 and can be checked from the ground truth data, where the centroid of the iris region is always located inside the region defined by the pupil. Once the centroid of the iris is computed, we force the only regional minimum of the image to be located at that point using morphological minima imposition. By this operation, the pupil region is emphasized and the pixel intensities beyond the pupil boundary are homogenized (see Figure 6a).

To filter noise, the image is converted to a binary one, and elementary erosion followed by elementary dilation, i.e. morphological opening (see Figure 6b), is applied on the binary image. Connected regions are extracted and the region with the greatest area is labelled as the pupil region. As in the outer border stage, the convex hull of the selected region is computed and a pixel mask that separates the pixels lying inside and outside the pupil border is created (Figure 6c).

The final ROI segmentation representing the bovine iris is given by the pixels lying inside the iris outer border (Figure 7a) minus the pixels lying inside the iris inner border (Figure $7 \mathrm{~b}$ ). The predicted ROI is contrasted with the ground truth ROI in Figure 7c.

\section{The BovineAAEyes80 dataset}

A big challenge in automated iris recognition systems is to capture a high-quality image of the iris since performance 


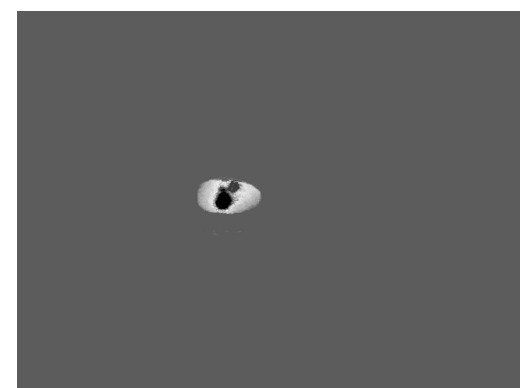

(a)

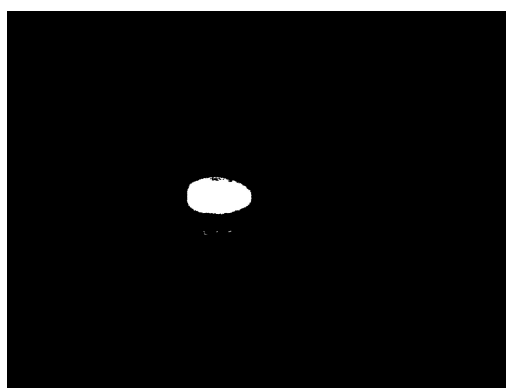

(b)

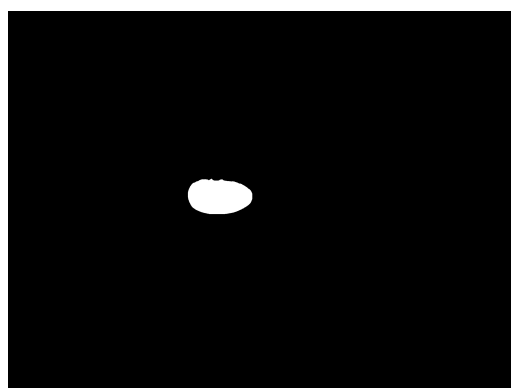

(c)

Figure 6: (a) Minima imposition result, (b) mask after morphological opening, (c) final pupil segmentation mask.

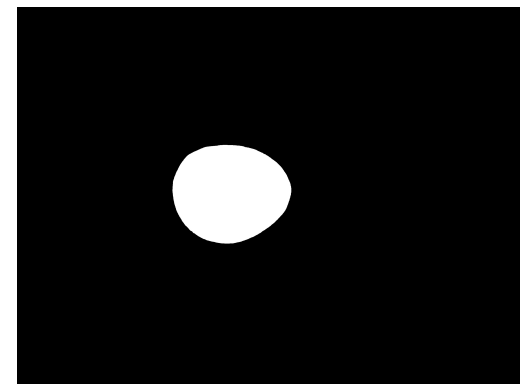

(a)

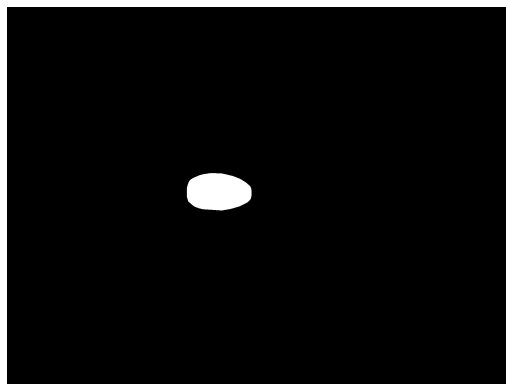

(b)

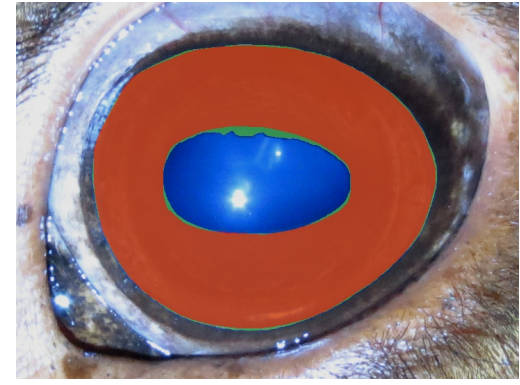

(c)

Figure 7: (a) Outer border region prediction, (b) Inner border region prediction, and (c) zoomed in ROI prediction (in red) contrasted with ground truth ROI (in green).

is strongly affected by poor-quality imaging [56]. This becomes even more critical in the case of non-cooperative behaviour. Our BovineAAEyes80 dataset comprises 80 RGB images of 11 different animals belonging to AberdeenAngus breed, the most diffused cattle breed in countries like the Argentine Republic [65] and the United States of America [66]. More specifically, six of the animals are Red Angus and five are Black Angus. For each image, an ID number unique for the animal is given.

Because of the non-cooperative behaviour of bovines [55], for practical reasons, these images have been taken from a cautious position. Ethical guidelines [67] have been taken into account during the acquisition, and all efforts were made to avoid animal suffering. [68].

Each image shows the bovine's head pictured from the left or right side. The eye and consequently the iris ROI does not necessarily cover most of the image and it can be at an arbitrary position. Unlike [55, 56], that uses an ad-hoc device, the photos have been taken at night using a Canon PowerShot SX4O HS regular visible light camera, employing the camera's built-in flash. The usage of flash is motivated by the need for recognition systems to work indoors, where artificial illumination is not sufficient to provide a good level of details in the images. In turn, a full recognition system is normally installed indoors, where the arti- ficial lighting conditions can often be not enough to guarantee the capture of enough details in the scene for the application under consideration. The capture distance from the camera to the animal varies between $100 \mathrm{~cm}$ and 200 $\mathrm{cm}$. The image resolution is $2100 \times 1575$ pixels for all the images composing the dataset. Each photo is labelled as $<$ animal number>_<image number>, with animal number ranging from 1 to 11, and image number starting at 1 for each different bovine.

For each image, a binary ground truth mask with white pixels belonging to the iris region has been manually created by two persons using photo-editing software. All the images have been independently labelled and the intersection of the two independent masks for each image was taken as the ground truth mask. Additionally, ground truth masks for the region surrounded by the outer border and the region surrounded by the inner border have been included for a more precise evaluation.

The dataset is publicly available online. ${ }^{1}$

$1 \mathrm{https}$ //juanilarregui.github.io/BovineAAEyes80 


\section{Experimental setup}

This Section describes the evaluation metrics that have been used during the experiments. Technical details about the hardware/software setup and the configurations of bilevel thresholding are also given.

Experiments have been conducted in a PC with a 4.00 GHz AMD FX-8350 eight-core processor CPU and 16 GB RAM. The code has been implemented by using MATLAB 2016a and it has been executed on a Windows 10 64-bits operating system.

\subsection{Evaluation metrics}

In our experiments, each image has been processed and the predicted mask has been compared with the ground truth data.

In particular, we define:

- A pixel correctly labelled as belonging to the iris region is considered as a true positive (TP);

- A pixel correctly labelled as not belonging to the iris region is considered as a true negative (TN);

- A pixel wrongly labelled as belonging to the iris region is considered as a false positive (FP);

- A pixel wrongly labelled as not belonging to the iris region is considered as a false negative (FN).

For the evaluation of the segmentation performance, we employ four different metrics, namely: Precision, Recall, F-score (F1) and Intersection over Union (IoU) [69]. None of these metrics takes into account the number of true negative cases. Instead, they focus on the positive cases of both the ground truth and the segmentation masks, that is, pixels that represent the iris region. Metrics that do consider true negative cases are in general not well suited for binary segmentation problems, especially when the ground truth mask does not cover most of the image since they give the same weight to pixels correctly identified as background and those correctly identified as part of the ROI.

The metrics are formally defined as follows:

$$
\begin{gathered}
\text { Precision }=\frac{T P}{T P+F P} \\
\text { Recall }=\frac{T P}{T P+F N} \\
\text { F-Score }=F_{1}=\frac{2}{\frac{1}{\text { Precision }} \cdot \frac{1}{\text { Recall }}}=\frac{2 T P}{2 T P+F P+F N} \\
\text { IoU }=\frac{T P}{T P+F P+F N}
\end{gathered}
$$

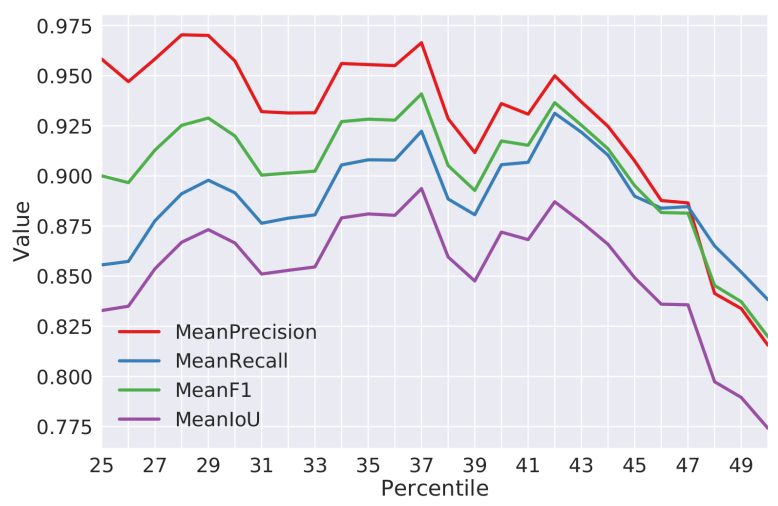

Figure 8: Different results of the proposed metrics varying the percentile for the bilevel threshold.

Precision and Recall are also called Positive Predictive Value and True Positive Rate, respectively. Intersection over Union represents the intersection of the computed segmentation and the ground truth areas, divided by their union. The F-score metric is the harmonic average of Precision and Recall. It can be interpreted as a metric comparable to IoU, with lower penalization for incorrect pixel classification.

From the equations, it can be observed that the F-Score and IoU metrics are related by:

$$
\frac{F_{1}}{2} \leq I o U \leq F_{1}
$$

All the aforementioned metrics take values in the range $[0,1]$, with 0 representing the lowest possible performance and 1 the highest.

\subsection{Bilevel threshold}

The optimal threshold for the gradient image has been found through a series of experiments at different values. Given the distribution of gradient magnitudes, threshold values for the experiments have been set as the percentiles between the first and the second quartiles, that is, all integer values between the 25th and the 50th percentiles. We tested the performance with the metrics described in Section 6.1. For each percentile, each metric included is the mean of that metric for all images. The output is shown in Figure 8, where Precision, Recall, F-score and IoU have been computed for different values of percentiles (x-axis). It is possible to observe that the maximum score for all the metrics but Precision is achieved setting the threshold at the 37th percentile, which has been chosen as the optimal value. 


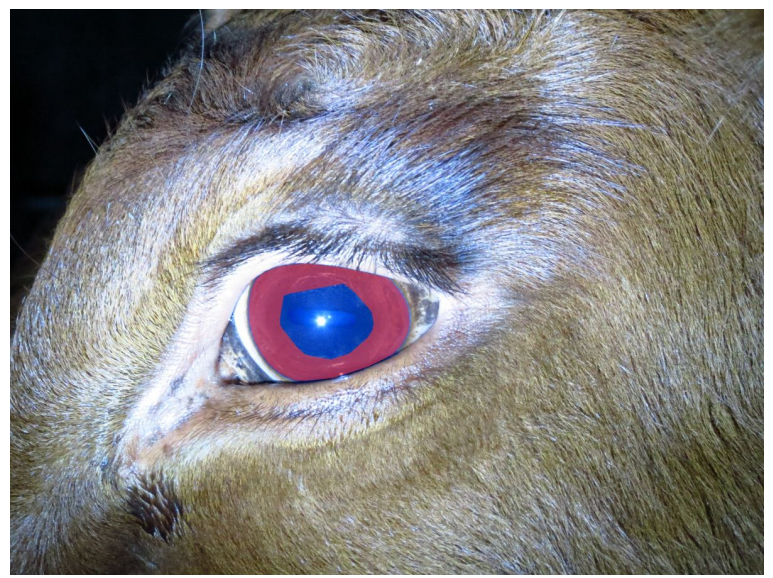

Figure 9: Inner border segmentation failure case.

\section{Experimental results and discussion}

The proposed solution has been tested with all images composing the BovineAAEyes 80 dataset with the metrics described in Section 6.1. Obtained results with single images composing the dataset are shown in Table 1; the last line reports results averaged over the entire dataset. The segmentation results have been reported by analyzing the performance of the overall segmentation procedure and, additionally, the evaluation of the two segmentation stages of the algorithm, namely, the outer and inner borders segmentation.

Considering the result on the most pessimistic metric, i.e. intersection over union, a mean score of 0.8957 has been achieved, while for the outer and inner borders segmentation stages the final scores are 0.9625 and 0.7619 , respectively.

In some images, the strong presence of the flash of the camera caused a massive blurring on the frontier between the iris and the pupil. The homogeneity of the pixel values around this border makes its identification more difficult in both RGB and HSV representations. In those cases, the method struggled to correctly segment the inner border, as can be seen in Figure 9. This explains the low precision and high recall cases in Table 1 for the Inner Border Stage. On the other hand, the area derived from the morphological minimum imposition and the opening operation, in some cases, does not reach the frontier between iris and pupil, resulting in a deficient segmentation with high precision but low recall values. These two reasons explain the degradation in the performance of the method for the pupil segmentation, especially compared to the metrics for the segmentation of the outer border.
Table 2 reports a comparison with state of the art methods. As we highlighted, other methods do not use a public dataset, do not provide an open source implementation and/or consider different input images, that are instead acquired with special hardware. Nevertheless, it shows how the proposed method works without constraints for the acquisition procedure and that, to the best of our knowledge, the first formal evaluation with a dataset that consists of images taken in real scenarios has been provided. The achieved preliminary results are really encouraging for building a fundamental block of a non-invasive bovine recognition system.

About performance, with the proposed solution each image is fully processed on an average time of 6.27 seconds in the aforementioned configuration.

\subsection{Comparison against human-oriented iris segmentation methods}

Taking into account that, to the best of our knowledge, no existing work deals with the scenario under consideration and that there are no open-source bovine eye segmentation algorithms nor datasets, we compare our method against well-proven algorithms for human iris segmentation, namely, Daugman's algorithm [70] for the segmentation of the inner and outer borders, and the algorithm proposed by Leo et al. [71], a method for the segmentation of the iris region. About the latter, it has been designed to exploit geometrical properties of isophotes in the image to select the most meaningful edge pixels and to classify them in subsets of equal isophote curvature, iteratively applying the method of De Marco et al. [72] for generic circle detection to segment the inner and outer border. With the dataset under consideration, the inner iris border completely failed due to the presence of flash or since the first outer border segmentation stage was already missing relevant parts of the pupil. Thus, the work in [71] has been tested only for the outer border. Table 3 shows global metrics, averaged over the entire dataset, and the score achieved by the best segmentation of each method. In both cases, the metric employed is Intersection over Union.

Figure 10 shows the best segmentation for each human-oriented method, illustrating the differences in the required approaches for correct human and bovine iris segmentation, and highlighting the difficulties that impose the non-circular nature of the bovine iris and pupil. 
Table 1: Obtained results with the BovineAAEyes 80 dataset.

\begin{tabular}{|c|c|c|c|c|c|c|c|c|c|c|c|c|}
\hline \multirow[b]{2}{*}{ Image } & & Overall $\mathrm{r}$ & ethod & & & Duter bo & r stage & & & Inner bo & r stage & \\
\hline & Precision & Recall & F1 & IoU & Precision & Recall & F1 & $\mathrm{IoU}$ & Precision & Recall & F1 & IoU \\
\hline L_1 & 0.9985 & 0.9350 & 0.9657 & 0.9337 & 0.9987 & 0.9590 & 0.9784 & 0.9578 & 0.8923 & 1.0000 & 0.9431 & 0.8923 \\
\hline 2 & 0.9991 & 9067 & 0.9507 & 0.9060 & 9992 & 0.9637 & .9812 & 9630 & 7213 & 1.0000 & & .7213 \\
\hline 3 & 0.9455 & & 0.9200 & .8519 & & & .9718 & .9451 & 5552 & & & 0.5552 \\
\hline 4 & 0.9998 & 421 & 0.9701 & 0.9419 & & & .9954 & .9908 & .7016 & & & 0.7016 \\
\hline 1 & 0.9908 & 9205 & 0.9544 & 0.9127 & .9996 & 9837 & .9916 & .9833 & .7202 & 0.9507 & & 0.6943 \\
\hline 2 & 0.9994 & 6734 & 0.8046 & 0.6731 & .9997 & 9840 & .9918 & 9837 & 2605 & 1.0000 & & .2605 \\
\hline${ }_{3} 3$ & 0.9938 & 0.6947 & 0.8178 & 0.6917 & & & & 0.9870 & & & & \\
\hline 4 & & 0.6678 & & 0.6646 & & & & 0.9856 & & & & \\
\hline _1 & & 8 & 0.97 & 0.94 & & & & 0.9734 & & & & \\
\hline 2 & & 30 & 0.9684 & 0.93 & 0.9973 & & & 0.9664 & & 0.9933 & & \\
\hline 3 & & 49 & 0.9698 & 0.9 & & & & 0.9676 & & 0.9992 & & .9156 \\
\hline-4 & 0.9965 & 9467 & 0.9710 & 0.94 & & & 855 & 0.9714 & & & & \\
\hline 5 & 0.9873 & 0.9543 & 0.9705 & 0.9427 & & 56 & 0.9791 & 0.9590 & & 0.9795 & & 9583 \\
\hline _6 & 0.9959 & 0.9523 & & 0.9485 & & & & 0.9721 & & & & \\
\hline 3_7 & & 0.9442 & & 0.9418 & & & & 0.9737 & & & & \\
\hline 8 & & & 0.96 & & & & & 71 & & & & \\
\hline 9 & & 0.9692 & 0.97 & 0.95 & & & & 0.97 & & & & \\
\hline 10 & & 9748 & 0.98 & 0.96 & & & & 0.9754 & 796 & 908 & & \\
\hline-1 & & 9693 & & 0.9 & & & & 732 & & & & \\
\hline 1 & 0. & 10 & 0.9625 & 0.9277 & & & & 0.97 & & & & \\
\hline 2 & & 0.88 & & & & & & & & & & \\
\hline 3 & & & & & & & & & & & & \\
\hline-4 & & & & & & & & & & & & \\
\hline-5 & & 94 & 0.97 & 0.94 & & & & 32 & & 877 & & \\
\hline _6 & & 9794 & 0.9409 & 0.88 & & & & 28 & & 786 & & \\
\hline 7 & & 72 & 9829 & 64 & & & & 34 & & & & \\
\hline 8 & & 14 & 0.9668 & & & & & & & & & \\
\hline _9 & & 0.9826 & 0.9553 & & & & & & & & & \\
\hline 10 & & & & & & & & & & & & \\
\hline 1 & & & & & & & & & & & & \\
\hline 1 & & 0.9541 & 0.9759 & 0.95 & & & & 0.9767 & & 955 & & \\
\hline _13 & 0 & 0.9521 & 0.9722 & 0.9 & & 50 & 319 & 9645 & & 759 & & \\
\hline _1 & & & & & & & & & & & & \\
\hline 5_2 & 0 & 0.943 & 0.97 & 0.94 & & 31 & & 0.9 & & & & \\
\hline $5 \_3$ & & & 0.9746 & & & & & & & & & \\
\hline $5 \_4$ & & & & & & & & & & & & \\
\hline 5 & & & 0.97 & & & & & & & & & \\
\hline 6 & & .9405 & 0.9689 & 0.93 & & & 788 & & 697 & 985 & & \\
\hline 5 & 9953 & 9311 & 0.9622 & 0.92 & 976 & 9505 & 735 & 83 & & 942 & & \\
\hline 1 & & & & & & & & & & & & \\
\hline $6 \_2$ & 0. & 0.9563 & 0.9639 & & & & & & & & & \\
\hline $6 \_3$ & & & & & & & & & & & & \\
\hline 5 & & & & & & & & & & & & \\
\hline 5 & & & & & & & & & & & & \\
\hline 6 & 0 & 9515 & 0.93 & 0.8791 & & & 784 & & & 4471 & & \\
\hline 1 & & & 0.72 & 0.56 & & & 606 & & & & & \\
\hline 1 & & & & & & & & & & & & \\
\hline 8_2 & & & & & & & & & & & & \\
\hline 8_3 & & & & & & & & & & & & \\
\hline-4 & & & & & & & & & & & & \\
\hline 5 & 0 & 9901 & 0.9172 & 0.84 & & & & & & & & \\
\hline -1 & & 646 & 0.7217 & 0.56 & & & 874 & & & & & \\
\hline 2 & & & & & & & 916 & & & & & \\
\hline 9_3 & & & & & & & 9764 & & & & & \\
\hline 9_4 & & & & & & & & & & & & \\
\hline 9_5 & & & & & & & & & & & & \\
\hline 6 & & & & & & & & & & & & \\
\hline 7 & & & 7 & & & & & & & & & \\
\hline 0 & 0 & 525 & 0.9754 & 0.95 & & 341 & 919 & & & 9989 & & \\
\hline P & & & & & & & & & & & & \\
\hline 10 & & & 0.9698 & & & 828 & 913 & & & 988 & & \\
\hline $10 \_4$ & & & & & & & & & & & & \\
\hline $10 \_5$ & & & & & & & & & & & & \\
\hline 0 & & & & & & & & & & & & \\
\hline & & & & & & & & & & & & \\
\hline & & & & & & & & & & & & \\
\hline & & & & & & & & & & & & \\
\hline 10_10 & 0. & 9565 & 0.9775 & 0.9559 & & 9858 & 0.9927 & & & 9988 & & 0.9010 \\
\hline 10_11 & & & & & & & & & & & & \\
\hline 11_1 & & & & & & & & & & & & \\
\hline & & & & & & & & & & & & \\
\hline & & & & & & & & & & & & \\
\hline & & & & & & & & & & & & \\
\hline & & & & & & & & & & & & \\
\hline 11_6 & 0.9424 & 0.9785 & 0.9601 & 0.9232 & & 0.9862 & 0.9847 & 0.9698 & 9986 & 0.9317 & 9640 & 0.9305 \\
\hline 11_7 & 0.9771 & 0.9626 & 0.9698 & 0.9414 & 0.9972 & 0.9769 & 0.9869 & 0.9742 & 0.9954 & 0.9645 & 0.9797 & 0.9602 \\
\hline 11 & & & & & & & & & & & & \\
\hline & & & & & & & & & & & & \\
\hline & & & & & & & & & & & & \\
\hline & 0.9939 & 0.9643 & 0.9789 & 0.9586 & 0.9981 & 0.9831 & 0.9905 & 0.9812 & 0.9699 & 0.9919 & 0.9808 & 0.9623 \\
\hline & & & & & & & & & & & & \\
\hline
\end{tabular}


Table 2: Comparison with state of the art methods.

\begin{tabular}{|c|c|c|c|c|}
\hline Method & Type of solution & Dataset description & $\begin{array}{l}\text { Acquisition hard- } \\
\text { ware }\end{array}$ & Results \\
\hline Zhang and Zhao [52] & $\begin{array}{l}\text { Circle fitting of } \\
\text { inner and outer } \\
\text { boundaries. }\end{array}$ & $\begin{array}{l}\text { Low-resolution images, } \\
\text { not publicly available. } \\
\text { No ground truth data for } \\
\text { segmentation. Bovine eye } \\
\text { occupying most of the } \\
\text { image. }\end{array}$ & NIR ad-hoc device. & $\begin{array}{l}\text { Only qualitative seg- } \\
\text { mentation results. }\end{array}$ \\
\hline Sun and Zhao [53] & $\begin{array}{l}\text { Inner segmenta- } \\
\text { tion and partial } \\
\text { outer segmenta- } \\
\text { tion. }\end{array}$ & $\begin{array}{l}\text { Low-resolution images, } \\
\text { not publicly available. } \\
\text { No ground truth data for } \\
\text { segmentation. Bovine eye } \\
\text { occupying most of the } \\
\text { image. }\end{array}$ & NIR ad-hoc device. & $\begin{array}{l}\text { No segmentation re- } \\
\text { sults, only overall } \\
\text { recognition (animal } \\
\text { identification) error. }\end{array}$ \\
\hline Lu et al. [55] & $\begin{array}{l}\text { Ellipse fitting of } \\
\text { inner and outer } \\
\text { boundaries. }\end{array}$ & $\begin{array}{l}60 \text { greyscale low- } \\
\text { resolution images from } \\
6 \text { animals, not publicly } \\
\text { available. No ground truth } \\
\text { data for segmentation. } \\
\text { Bovine eye occupying } \\
\text { most of the image. }\end{array}$ & NIR ad-hoc device. & $\begin{array}{l}\text { Metrics as qualitatively } \\
\text { correct segmetations. } \\
\text { Inner boundary Accu- } \\
\text { racy: } 96.00 \% \text {, Outer } \\
\text { boundary Accuracy: } \\
76.70 \% \text {. }\end{array}$ \\
\hline Larregui et al. [57] & $\begin{array}{l}\text { Partial segmenta- } \\
\text { tion of inner and } \\
\text { outer boundaries. }\end{array}$ & $\begin{array}{l}48 \text { RGB mid-resolution } \\
\text { images from } 8 \text { animals, } \\
\text { not publicly available. } \\
\text { No ground truth data for } \\
\text { segmentation. Bovine eye } \\
\text { does not occupy most of } \\
\text { the image. }\end{array}$ & $\begin{array}{l}\text { Regular visible } \\
\text { light camera. }\end{array}$ & $\begin{array}{l}\text { Metric as qualitatively } \\
\text { correct segmenta- } \\
\text { tions. Overall Accuracy: } \\
91.67 \% \text {. }\end{array}$ \\
\hline Proposed & $\begin{array}{l}\text { Complete segmen- } \\
\text { tation of inner and } \\
\text { outer boundaries. }\end{array}$ & $\begin{array}{l}80 \text { RGB high-resolution } \\
\text { images from } 11 \text { ani- } \\
\text { mals, publicly available. } \\
\text { Includes ground truth } \\
\text { masks for segmentation. } \\
\text { Bovine eye does not } \\
\text { occupy most of the image. }\end{array}$ & $\begin{array}{l}\text { Regular visible } \\
\text { light camera. }\end{array}$ & $\begin{array}{l}\text { Quantitative metrics, } \\
\text { computed against } \\
\text { ground truth masks. } \\
\text { Overall mean loU: } \\
89.57 \% \text {, Inner bound- } \\
\text { ary mean loU: } 76.19 \% \text {, } \\
\text { Outer boundary mean } \\
\text { loU: } 96.25 \% \text {. }\end{array}$ \\
\hline
\end{tabular}

Table 3: Comparison against human-oriented methods by Intersection over Union metric.

\begin{tabular}{lcccccc}
\hline & \multicolumn{3}{c}{ Mean loU } & \multicolumn{3}{c}{ Best case loU } \\
\cline { 2 - 7 } Method & Overall method & Outer stage & Inner stage & Overall method & Outer stage & Inner stage \\
\hline Daugman [70] & 0.0706 & 0.4177 & 0.1468 & 0.2037 & 0.7047 & 0.2421 \\
Leo et al. [71] & - & 0.5187 & - & - & 0.7715 & - \\
Proposed & $\mathbf{0 . 8 9 5 7}$ & $\mathbf{0 . 9 6 2 5}$ & $\mathbf{0 . 7 6 1 9}$ & $\mathbf{0 . 9 6 6 4}$ & $\mathbf{0 . 9 9 0 8}$ & $\mathbf{0 . 9 7 9 8}$ \\
\hline
\end{tabular}




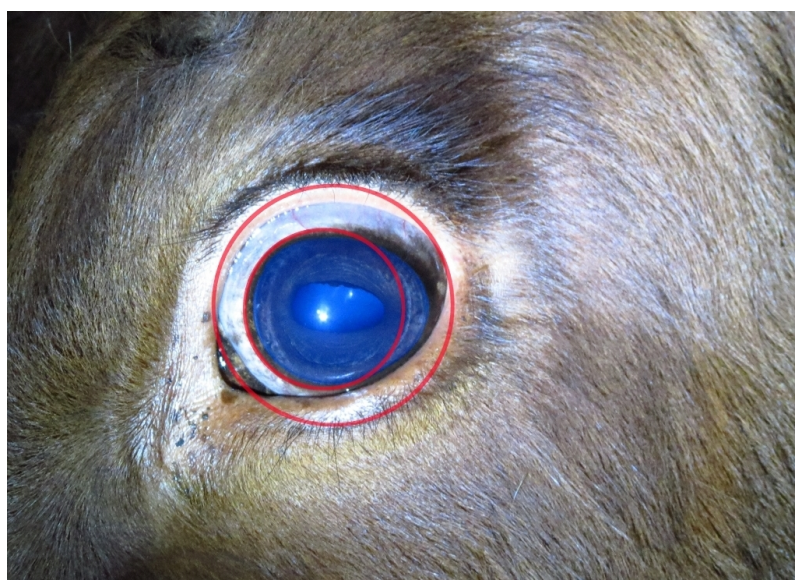

(a)

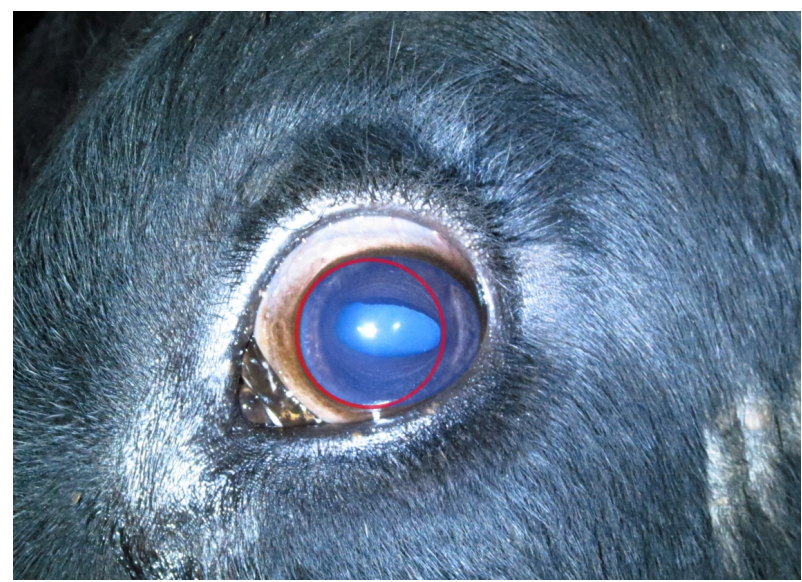

(b)

Figure 10: (a) Best segmentation by Daugman's algorithm [70], (b) best segmentation with Leo et al. [71] (outer border).

\section{Conclusions}

Most employed methods for animal identification represent invasive solutions that cannot ensure adequate security or resistance to tampering. In this work, a non-invasive algorithm for bovine iris segmentation has been proposed. The main advantage of the solution is that it can deal with images taken in the wild with regular visible-light cameras, with potential industrial applications to real scenarios, overcoming the limitations of the state of the art in terms of ad-hoc expensive hardware or position of the eye in the image. To the best of our knowledge, this is the first work on bovine iris segmentation with images taken in the wild, without imposing the state of the art constraints and coming with an evaluation method based on comparison with a dataset composed of bovine iris images and manually labelled ground truth data, made publicly available; this way, the gap of a formal algorithmic evaluation on a specific dataset with ground truth information has been filled. The dataset has been designed for assessing iris recognition and cow identification purposes, presenting both ground truth data and identification number for each animal. Future works will investigate the possibility of improving the achieved performance. Moreover, algorithms for the final block of an automatic bovine identification system will be investigated in order to provide a complete automatic solution in different countries where such a system could be adopted. Finally, further studies about the feasibility of the proposed solution to be applied to the segmentation problem in other cattle breeds will be conducted.

\section{References}

[1] Bridle J., Automatic dairy cow identification, Journal of Agricultural Engineering Research, 21(1), 1976, 41-48

[2] Cambier J.L., System and method for animal identification using IRIS images, 2012, uS Patent 8,189,879

[3] Shadduck J., Golden B., Retinal imaging in secure identification and source verification of livestock, Proceedings ID/INFO Expo, 2002

[4] Kumar S., Tiwari S., Singh S.K., Face recognition for cattle, in 2015 Third International Conference on Image Information Processing (ICIIP), IEEE, 2015, 65-72

[5] Evans J., Van Eenennaam A., Livestock identification, Emerging management systems in animal identification. Fact Sheet, 5, 2005

[6] Bai H., Zhou G., Hu Y., Sun A., Xu X., Liu X., Lu C., Traceability technologies for farm animals and their products in China, Food Control, 79, 2017, 35-43

[7] Hammer N., Pfeifer M., Staiger M., Adrion F., Gallmann E., Jungbluth T., Cost-benefit analysis of an UHF-RFID system for animal identification, simul taneous detection and hotspot monitoring of fattening pigs and dairy cows, LANDTECHNIK, 72(3), 2017, 130-155

[8] Marchant J., Secure animal identification and source verification, JM Communications, UK. Copyright Optibrand Ltd., LLC, 2002

[9] Deiner K., Bik H.M., Mächler E., Seymour M., LacoursièreRoussel A., Altermatt F., Creer S., Bista I., Lodge D.M., de Vere N., et al., Environmental DNA metabarcoding: transforming how we survey animal and plant communities, Molecular ecology, 26(21), 2017, 5872-5895

[10] Huang J., Wang Y., Tan T., Cui J., A new iris segmentation method for recognition, In Proceedings of the 17th International Conference on Pattern Recognition, 2004. ICPR 2004., volume 3, IEEE, 2004, 554-557

[11] Malisiewicz T., Efros A.A., Improving Spatial Support for Objects via Multiple Segmentations., In N.M. Rajpoot, A.H. Bhalerao, eds., BMVC, British Machine Vision Association, 2007, 1-10 
[12] Rabinovich A., Vedaldi A., Belongie S., Does image segmentation improve object categorization, Technical report, 2007

[13] Witten I.H., Frank E., Hall M.A., Pal C.J., Data Mining: Practical machine learning tools and techniques, Morgan Kaufmann, 2016

[14] Daugman J., How iris recognition works, IEEE Transactions on circuits and systems for video technology, 14(1), 2004, 21-30

[15] Lu Ming, Image segmentation algorithm research and improvement, In 2010 3rd International Conference on Advanced Computer Theory and Engineering(ICACTE), volume 5, 2010, V5-211V5-214, 10.1109/ICACTE.2010.5579114

[16] Kumar S., Singh S.K., Visual animal biometrics: survey, IET Biometrics, 6(3), 2017, 139-156, 10.1049/iet-bmt.2016.0017

[17] Duyck J., Finn C., Hutcheon A., Vera P., Salas J., Ravela S., Sloop: A pattern retrieval engine for individual animal identification, Pattern Recognition, 48(4), 2015, 1059 - 1073, https://doi.org/10.1016/j.patcog.2014.07.017

[18] Varior R.R., Haloi M., Wang G., Gated Siamese Convolutional Neural Network Architecture for Human Re-Identification, CoRR, abs/1607.08378, 2016

[19] Raghavendra C., Kumaravel A., Sivasubramanian S., Iris technology: A review on iris based biometric systems for unique human identification, In Algorithms, Methodology, Models and Applications in Emerging Technologies (ICAMMAET), 2017 International Conference on, IEEE, 2017, 1-6

[20] He Z., Tan T., Sun Z., Qiu X., Toward accurate and fast iris segmentation for iris biometrics, IEEE transactions on pattern analysis and machine intelligence, 31(9), 2009, 1670-1684

[21] Mei J., Si Y., Gao H., Novel approaches to improve robustness accuracy and rapidity of iris recognition systems, IEEE transactions on industrial informatics, 8(1), 2011, 110-117

[22] Hofbauer H., Alonso-Fernandez F., Bigun J., Uhl A., Experimental analysis regarding the influence of iris segmentation on the recognition rate, IET Biometrics, 5(3), 2016, 200-211

[23] Hajari K., Bhoyar K., A review of issues and challenges in designing iris recognition systems for noisy imaging environment, In Pervasive Computing (ICPC), 2015 International Conference on, IEEE, 2015, 1-6

[24] Nigam I., Vatsa M., Singh R., Ocular biometrics: A survey of modalities and fusion approaches, Information Fusion, 26, 2015, 1 - 35, https://doi.org/10.1016/j.inffus.2015.03.005

[25] Ahmadi N., Akbarizadeh G., Hybrid robust iris recognition approach using iris image pre-processing, two-dimensional gabor features and multi-layer perceptron neural network/PSO, IET Biometrics, 7(2), 2017, 153-162

[26] Arslan A., Şen B., Çelebi F.V., Uysal B.S., Automatic segmentation of region of interest for dry eye disease diagnosis system, in Signal Processing and Communication Application Conference (SIU), 2016 24th, IEEE, 2016, 1817-1820

[27] De Marsico M., Petrosino A., Ricciardi S., Iris recognition through machine learning techniques: A survey, Pattern Recognition Letters, 82, 2016, 106-115

[28] Ramlee R., Ramli A., Noh Z., Pupil Segmentation of Abnormal Eye using Image Enhancement in Spatial Domain, In Materials Science and Engineering Conference Series, volume 210, 2017, 012031

[29] Telgad R., Siddiqui A., Deshmukh P., Survey: Iris feature extraction techniques for persons recognition system, Advances in Computational Research, 7(1), 2015, 176
[30] Sankowski W., Grabowski K., Napieralska M., Zubert M., Napieralski A., Reliable algorithm for iris segmentation in eye image, Image and vision computing, 28(2), 2010, 231-237

[31] Jeong D.S., Hwang J.W., Kang B.J., Park K.R., Won C.S., Park D.K., Kim J., A new iris segmentation method for non-ideal iris images, Image and vision computing, 28(2), 2010, 254-260

[32] Gou C., Wu Y., Wang K., Wang K., Wang F.Y., Ji Q., A joint cascaded framework for simultaneous eye detection and eye state estimation, Pattern Recognition, 67, 2017, 23-31

[33] Hansen D.W., Ji Q., In the eye of the beholder: A survey of models for eyes and gaze, IEEE transactions on pattern analysis and machine intelligence, 32(3), 2010, 478-500

[34] Zhang X., Sugano Y., Fritz M., Bulling A., MPIIGaze: Real-World Dataset and Deep Appearance-Based Gaze Estimation, IEEE Transactions on Pattern Analysis and Machine Intelligence, 2018, 1-1, 10.1109/TPAMI.2017.2778103

[35] Wang P., Green M.B., Ji Q., Wayman J., Automatic eye detection and its validation, In Computer Vision and Pattern RecognitionWorkshops, 2005. CVPR Workshops. IEEE Computer Society Conference on, IEEE, 2005, 164-164

[36] Valenti R., Gevers T., Accurate eye center location and tracking using isophote curvature, In Computer Vision and Pattern Recognition, 2008. CVPR 2008. IEEE Conference on, IEEE, 2008, 1-8

[37] Leo M., Cazzato D., De Marco T., Distante C., Unsupervised approach for the accurate localization of the pupils in nearfrontal facial images, Journal of Electronic Imaging, 22(3), 2013, 033033-033033

[38] Leo M., Cazzato D., De Marco T., Distante C., Unsupervised eye pupil localization through differential geometry and local selfsimilarity matching, PloS one, 9(8), 2014, e102829

[39] Valenti R., Gevers T., Accurate eye center location through invariant isocentric patterns, IEEE Transactions on Pattern Analysis and Machine Intelligence, 34(9), 2012, 1785-1798

[40] Fuhl W., Kübler T., Sippel K., Rosenstiel W., Kasneci E., Excuse: Robust pupil detection in real-world scenarios, In International Conference on Computer Analysis of Images and Patterns, Springer, 2015, 39-51

[41] Borza D., Darabant A.S., Danescu R., Real-Time Detection and Measurement of Eye Features from Color Images, Sensors, 16(7), 2016, 1105

[42] Fuhl W., Santini T., Kasneci G., Kasneci E., PupilNet: convolutional neural networks for robust pupil detection, arXiv preprint arXiv:1601.04902, 2016

[43] Allen A., Golden B., Taylor M., Patterson D., Henriksen D., Skuce R., Evaluation of retinal imaging technology for the biometric identification of bovine animals in Northern Ireland, Livestock Science, 116(1), 2008, 42-52

[44] Kumar S., Singh S.K., Singh R., Singh A.K., Animal Biometrics: Techniques and Applications, Springer, 2018

[45] Li W., Ji Z., Wang L., Sun C., Yang X., Automatic individual identification of Holstein dairy cows using tailhead images, Computers and Electronics in Agriculture, 142, 2017, 622-631

[46] Musgrave C., Cambier J.L., System and method of animal identification and animal transaction authorization using iris patterns, 2002, uS Patent 6,424,727

[47] Noviyanto A., Arymurthy A.M., Automatic cattle identification based on muzzle photo using speed-up robust features approach, In Proceedings of the 3rd European conference of computer science, ECCS, volume 110, 2012, 114 
[48] Lowe D.G., Object recognition from local scale-invariant features, in Computer vision, 1999. The proceedings of the seventh IEEE international conference on, volume 2, leee, 1999, 11501157

[49] Chen J., Shan S., He C., Zhao G., Pietikainen M., Chen X., Gao W., WLD: A robust local image descriptor, IEEE transactions on pattern analysis and machine intelligence, 32(9), 2010, 17051720

[50] He D.C., Wang L., Texture unit, texture spectrum, and texture analysis, IEEE transactions on Geoscience and Remote Sensing, 28(4), 1990, 509-512

[51] Gaber T., Tharwat A., Hassanien A.E., Snasel V., Biometric cattle identification approach based on weber's local descriptor and adaboost classifier, Computers and Electronics in Agriculture, 122, 2016, 55-66

[52] Zhang M., Zhao L., An iris localization algorithm based on geometrical features of cow eyes, in MIPPR 2009: Automatic Target Recognition and Image Analysis, volume 7495, International Society for Optics and Photonics, 2009, 749517

[53] Sun S., Zhao L., Bovine iris segmentation using region-based active contour model, International Journal of Innovative Computing, Information and Control, 8(9), 2012, 6461-6471

[54] Zhao L., Shengnan S., Wang X., Tracking and traceability system using livestock Iris code in meat supply chain, International Journal of Innovative Computing, Information and Control, 7(5), 2011, 2201-2212

[55] Lu Y., He X., Wen Y., Wang P.S., A new cow identification system based on iris analysis and recognition, International journal of biometrics, 6(1), 2014, 18-32

[56] He X., Yan J., Chen G., Shi P., Contactless autofeedback iris capture design, IEEE Transactions on Instrumentation and Measurement, 57(7), 2008, 1369-1375

[57] Larregui J.I., Espinosa J., Ganuza M.L., Castro S.M., Biometric Iris Identification in Bovines, in Computer Science \& Technology Series, XX Argentine Congress of Computer Science selected papers, 2015, 111-121

[58] Bora D.J., Gupta A.K., Khan F.A., Comparing the performance of $L^{\star} A^{\star} B^{\star}$ and HSV color spaces with respect to color image segmentation, arXiv preprint arXiv:1506.01472, 2015
[59] Sural S., Qian G., Pramanik S., Segmentation and histogram generation using the HSV color space for image retrieval, In Image Processing. 2002. Proceedings. 2002 International Conference on, volume 2, IEEE, 2002, II-II

[60] Soille P., Advances in the analysis of topographic features on discrete images, In Discrete Geometry for Computer Imagery, Springer, 2002, 271-296

[61] Vincent L., Morphological grayscale reconstruction in image analysis: Applications and efficient algorithms, IEEE transactions on image processing, 2(2), 1993, 176-201

[62] Meyer F., Topographic distance and watershed lines, Signal processing, 38(1), 1994, 113-125

[63] El Kaddouhi S., Saaidi A., Abarkan M., Eye detection based on the Viola-Jones method and corners points, Multimedia Tools and Applications, 76(21), 2017, 23077-23097

[64] Rosin P.L., Unimodal thresholding, Pattern recognition, 34(11), 2001, 2083-2096

[65] Angus es la raza predominante de la ganaderia argentina, http://www.infocampo.com.ar/angus-es-la-razapredominante-de-la-ganaderia-argentina/, [Online; Accessed: 2018-09-16]

[66] FAQs - American Angus Association, http://www.angus.org/ Pub/FAQs.aspx, [Online; Accessed: 2019-03-03]

[67] Kilkenny C., Browne W., Cuthill I.C., Emerson M., Altman D.G., Animal research: reporting in vivo experiments: the ARRIVE guidelines, British journal of pharmacology, 160(7), 2010, 15771579

[68] Directive E., DIRECTIVE 2010/63, EU of the European Parliament and of the Council of, 22, 2010

[69] Taha A.A., Hanbury A., Metrics for evaluating 3D medical image segmentation: analysis, selection, and tool, BMC medical imaging, 15(1), 2015, 29

[70] Daugman J.G., High confidence visual recognition of persons by a test of statistical independence, IEEE transactions on pattern analysis and machine intelligence, 15(11), 1993, 1148-1161

[71] Leo M., De Marco T., Distante C., Highly usable and accurate iris segmentation, in 2014 22nd International Conference on Pattern Recognition, IEEE, 2014, 2489-2494

[72] De Marco T., Cazzato D., Leo M., Distante C., Randomized circle detection with isophotes curvature analysis, Pattern Recognition, 48(2), 2015, 411-421 